\title{
Managing pain in sleep surgery patients
}

\section{Rishi Mandavia, Nishchay Mehta, Jim Roberts, Jamie Turnbull, Vik Veer}

Royal National Throat Nose and Ear Hospital

\section{Objectives}

- Sleep surgery patients typically experience significant post-operative pain, with subsequent impact on quality of life.

-There is uncertainty around optimal peri-operative analgesia strategies for these patients, with resultant variation in practices.

- This paper presents a literature review and our novel practice on analgesia strategies for patients undergoing sleep surgery.

\section{Methods}

Literature review:

- A literature search was performed using the Medline and Embase databases.

- An article was included if it discussed analgesia strategies for patients undergoing sleep surgery.

\section{Case series:}

- Using visual analogue pain scores we prospectively collected data on patients undergoing sleep surgery at our centre.

- We compared the mean VAS between 2 groups:

1) Novel peri-operative analgesia strategy:

- $0.3-0.5 \mathrm{mg} / \mathrm{kg}$ Ketamine, Paracetamol $1 \mathrm{~g}$ IV, Diclofenac $1 \mathrm{mg} / \mathrm{kg}$, Fentanyl $2 \mathrm{mcg} / \mathrm{kg}$ (up to $200 \mathrm{mcg}$ ), $0.05-0.10 \mathrm{mg} / \mathrm{kg}$ Morphine.

2) Previous perioperative analgesia strategy: - Diclofenac 75mg IV, Morphine 10mg IV, Paracetamol 1g IV, Fentanyl 100 mcg IV.

Patients were matched for their specific operation.

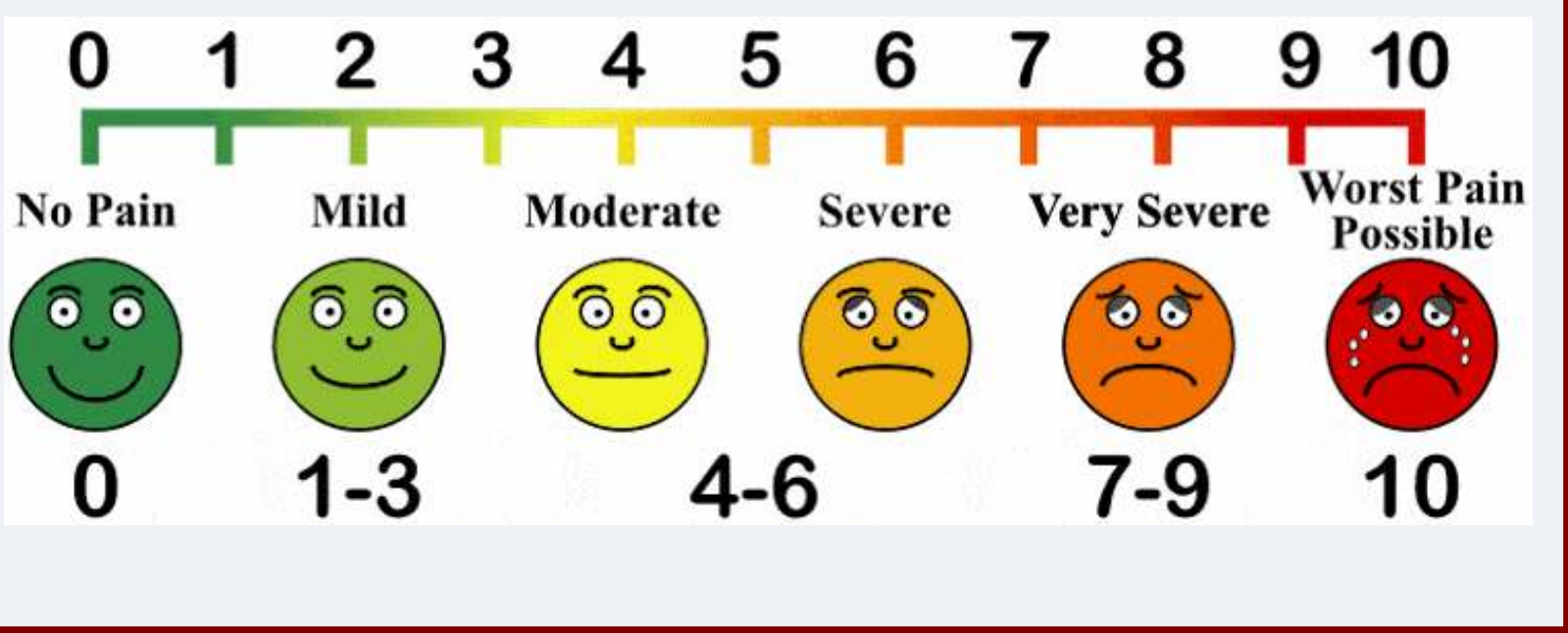

\section{Results}

Literature review:

- A wide range of peri-operative analgesia strategies were identified for patients undergoing sleep surgery. These broadly consisted of: 1) Opioids alone 2) Opioids with gabapentin 3) Opioids with ketorolac tromethamine.

- There was considerable heterogeneity in outcome measures to assess post-operative pain.

- There is uncertainty in the literature with regards to the optimal peri-operative analgesia strategy for patients undergoing sleep surgery.

\section{Case series:}

- Patient age ranged from $22-65$ years.

- 105 patients received our novel analgesia strategy.

- 168 patients received our previous analgesia strategy.

- Operations included: tonsillectomy, adenotonsillectomy, uvulopalatoplasty, palatoplasty, uvulopalatopharyngoplasty, expansion pharyngoplasty, RFA tongue base, RFA soft palate.

- Mean matched VAS pain score reduced by 3 when using our novel analgesia strategy.

\section{Conclusion}

- Our peri-operative analgesia strategy appears to result in reduced post-operative pain for patients undergoing surgery for sleep disordered breathing.

- Larger comparative studies are required to confirm our findings.

- We highlight the need for consensus on outcome measures for post-operative pain in sleep surgery. 